\title{
TAMAÑO DEL GOBIERNO Y BIENESTAR INDIVIDUAL EN PAÍSES DE LA ALIANZA DEL PACÍFICO
}

\section{SIZE OF GOVERNMENT AND INDIVIDUAL WELFARE IN COUNTRIES OF THE PACIFIC ALLIANCE}

\author{
Vladimir Rodríguez Cairo \\ Doctor en Derecho y Ciencia Política, Magister en Economía - Docente Principal de la Facultad de Ciencias Contables Universidad \\ Nacional Mayor de San Marcos, Lima, Perú - Email: vrodriguezc@unmsm.edu.pe (Autor Corresponsal)
}

[Recibido: 2016/12/14 Aceptado: 2017/02/20]

\begin{abstract}
RESUMEN
La investigación permitió determinar el grado de relación entre el tamaño del gobierno y el bienestar individual en los países miembros de la Alianza del Pacífico durante el período 1970-2014. A partir de dicho objetivo, se realizó un análisis descriptivo sobre la relación entre las variables y se utilizaron dos aproximaciones basadas en el análisis de regresión y la determinación del coeficiente de correlación. Siendo así, se obtuvieron como principales resultados que existe una relación muy positiva entre el tamaño del gobierno y el Producto Bruto Interno per cápita en el caso chileno, y menor en el caso de Perú, México y Colombia. De este modo, se concluyó que el tamaño del gobierno es uno de los factores que influyó en el bienestar individual, y consiguientemente, en el bienestar general de la población, pero constituye una condición necesaria pero no suficiente para determinar el bienestar.
\end{abstract}

PALABRAS CLAVE

Índice de libertad económica, intervención del gobierno, bienestar individual, alianza del pacífico.

\begin{abstract}
The research allowed to determine the degree of relationship between government size and individual welfare in the Pacific Alliance countries during the period 1970-2014. From this objective, a descriptive analysis was performed on the relationship between the variables and two estimates were used based on the regression analysis and the determination of the correlation coefficient. Thus, the main results obtained show a very positive relationship between the size of the government and the per capita gross domestic product in the Chilean case, and lower in the case of Peru, Mexico and Colombia. Therefore, it was concluded that government size is one of the factors that influenced individual well-being, and consequently, the general well-being of the population, but it is a necessary but not sufficient condition for determining welfare.
\end{abstract}

\section{KEYWORDS}

Index of economic freedom, government intervention, individual well-being, pacific alliance.

Como Citar: Rodríguez, V. (2017). Tamaño del gobierno y bienestar individual en países de la Alianza del Pacífico. Quipukamayoc, 25(48), 9-17. doi: http://dx.doi.org/10.15381/quipu.v25i48.13986

\section{INTRODUCCIÓN}

El estudio analiza la intervención del gobierno, específicamente el tamaño del gobierno en los países miembros de la Alianza del Pacífico a lo largo de las cuatro últimas décadas. Para tal efecto, se formula la siguiente inte- rrogante: ¿Cuál es el grado de relación entre el índice del tamaño del gobierno y el PBI per cápita en países miembros de la Alianza del Pacífico en el período 1970-2014?

En ese sentido, la hipótesis que responde a la pregunta es: El grado de correlación entre el índice del tamaño del gobierno como componente del Índice de libertad económica y el Producto Bruto Interno (PBI) per cápita en países miembros de la Alianza del Pacífico como Perú, México y Colombia es menor comparado con Chile, durante el período 1970-2014. 
Para tal efecto, se exponen los fundamentos teóricos de la intervención del gobierno de Ludwig von Mises y Murray Rothbard. También se plantea la base teórica del bienestar de Stiglitz, Sen y Fitoussi. Asimismo, se analizan los datos del tamaño del gobierno del Economic Freedom of the World 2016 Annual Report publicado por Fraser Institute de Canadá y del Producto Bruto Interno per cápita para países de la Alianza del Pacífico (AP).

En definitiva, la mayoría de estudios analizados permiten observar la relación entre libertad y crecimiento de diversos países, sin embargo, el presente estudio se enfoca en la relación particular que existe entre el tamaño del gobierno y el bienestar en los países de la AP. Dentro de este contexto, la intervención del gobierno termina afectando el bienestar individual a través de las restricciones en la libertad de elegir de los agentes económicos.

Bajo tal premisa, el estudio recurre al enfoque cuantitativo, por ser probatorio a través de las pruebas estadísticas de regresión y correlación. Por cierto, la principal limitación de la investigación es el número de observaciones disponibles, lo que restringe el uso de análisis estadísticos más avanzados. Dado que se cuenta con una serie anual de corta duración no se utilizan otras variables que podrían enriquecer el análisis para no perder grados de libertad.

Para una mejor comprensión del presente estudio, su contenido se ha estructurado en cuatro partes. La primera comprende las bases teóricas. En la segunda se plantea la metodología. La tercera abarcan los resultados. En la cuarta se aborda la discusión. Luego, se presentan las conclusiones. Finalmente, se da a conocer las referencias bibliográficas.

\section{Intervención del gobierno}

La intervención del gobierno es una discusión válida y relevante cuando se pretende analizar el grado de relación entre el tamaño del gobierno y el bienestar. Siendo así, de acuerdo con Mises (2011), la intervención es un decreto emanado directa o indirectamente de la autoridad que desempeña el poder administrativo de la sociedad y que obliga a los empresarios y capitalistas a explotar determinados factores de producción de forma distinta de como los explotarían si sólo tuvieran que obedecer a los dictados del mercado. Como es lógico, cuanto más amplía el poder público el ámbito de su acción, tanto más se hipertrofia el presupuesto. Los poderes públicos no tienen, por eso, otro remedio que acudir a las medidas tributarias. Para nutrir el presupuesto, han de exigir de los ciudadanos una porción de su respectivo patrimonio o renta (p. 869).

En línea con lo expresado por Mises (2011) el talón de Aquiles del mecanismo fiscal radica en la paradoja de que cuanto más se incrementan los impuestos, tanto más se debilita la economía de mercado y, consecuentemente, el propio sistema impositivo. El mantenimiento de la propiedad privada y las confiscatorias medidas fiscales resultan incompatibles ( $\mathrm{p}$. 874).

Definitivamente, es el intercambio en el mercado lo que nos permite precisamente ir elevando nuestro nivel de bienestar. Por ello, contrariamente a lo sostenido por Mises (2011), para Rothbard (2015, p. 106) lo más importante es que lo que buscan muchos economistas, un impuesto neutral es decir, un impuesto que mantenga el mercado igual que si no hubiera impuestos- será siempre una quimera. Ningún impuesto puede ser ver- daderamente neutral; todos causarán distorsiones. Solo se puede alcanzar la neutralidad en un mercado completamente libre, donde los ingresos gubernamentales solo se obtengan por compras voluntarias.

Desde tal perspectiva, Rothbard (2015) en su tratado sobre intervencionismo "Poder y mercado" realiza una tipología sistemática de la intervención del gobierno a las cuales considera ilegítimas. En estricto, denomina intervención autista, cuando el Estado obliga a un sujeto, sin recibir ningún bien o servicio a cambio (homicidio); binaria, cuando el Estado fuerza al sujeto a hacer un intercambio o un «regalo» unilateral de algún bien o servicio al invasor (impuestos o gasto público), y triangular, cuando este interfiere en la interacción entre dos partes (controles de precios o regulaciones de productos).

De ahí que para Rothbard (2015) resulta curioso que quienes escriben sobre economía política solo han reconocido a la tercera categoría como intervención (triangular). Cuestiona el hecho de que se desarrollen curiosos esquemas en los que el mercado se considera como absolutamente «libre»y sin estorbos, a pesar de que haya un sistema regulado de impuestos obligatorios (p. 13-14).

El autor añade que una agencia intervencionista, como el gobierno, debe gastar fondos, lo que en economía monetaria significa gastar dinero. Este dinero solo puede obtenerse de los ingresos. La mayoría de los ingresos $-\mathrm{y}$ la razón por la que llamamos intervencionista a la agencia- deben proceder de dos fuentes: en el caso del gobierno, los impuestos y la inflación. Los impuestos son una apropiación coercitiva, que el gobierno extrae del pueblo; la inflación es la emisión bá- 
sicamente fraudulenta de certificaciones o nuevo dinero Rothbard, 2015 (p. 101).

Por lo tanto, resulta importante la medición del tamaño del gobierno para comprender el grado de intervención en la economía. Desde luego, si el gobierno interviene significativamente en el mercado terminará a la larga distorsionando las decisiones de los agentes económicos, generando que los resultados no sean los más convenientes, $\mathrm{y}$ viceversa.

El otro argumento para la intervención del gobierno, contrario a lo expuesto en los párrafos precedentes, es la existencia de fallas del mercado. Sobre este enfoque, Stiglitz y Ronsengard (2016) consideran que el primer teorema fundamental de la economía del bienestar establece que la economía sólo es eficiente en el sentido de $\mathrm{Pa}$ reto en determinadas circunstancias o condiciones. Hay seis importantes condiciones en las que los mercados no son eficientes en el sentido de $\mathrm{Pa}$ reto. Se denominan fallas del mercado y constituyen un argumento a favor de la intervención del Estado (p. 125).

\section{Tamaño del gobierno}

Con bastante frecuencia se mide el grado de participación del gobierno en las actividades económicas comparando los ingresos y gastos públicos con el nivel de producción de una economía (Producto Bruto Interno - PBI). En esa orientación, Stiglitz y Rosengard (2016) sostienen que uno de los indicadores que resulta especialmente cómodo a los economistas es la magnitud del gasto público en relación con el conjunto de la economía. Un indicador habitual de la dimensión del conjunto de la economía es el PBI, que es una medida del valor de todos los bienes y servicios producidos en la economía durante un determinado año (p. 76).

Es así que los Ingresos del Estado están conformados por: a) Ingresos corrientes: impuestos, tasas, contribuciones, venta de bienes, prestación de servicios, multas; b) Ingresos de capital: venta de activos (inmuebles, terrenos, maquinarias, acciones del Estado en Empresas); c) Transferencias; d) Financiamiento: operaciones oficiales de crédito.

Por su parte, los Gastos del Estado se componen de: a) Gastos corrientes: personal y obligaciones sociales, obligaciones previsionales, bienes $y$ servicios; b) Gastos de capital: inversión pública; c) Servicio de la deuda: intereses y cargos de la deuda, amortización de la deuda.

En ese entender, la participación del Estado en la economía varía significativamente de un país a otro. Es así que países emergentes cobran impuestos alrededor del $15 \%$ de lo que produce en dicho país, mientras que en los países desarrollados los ingresos del gobierno son superiores al 25\% como porcentaje del nivel de producción.

No obstante lo señalado en los párrafos precedentes, otra forma de medir el tamaño del gobierno es según Gwartney, Lawson y Hall (2016) el Índice de Libertad Económica en el Mundo, el cual mide el grado en que

\section{Tabla 1.}

Componentes y subcomponentes de la libertad de comercio internacional Fuente: Gwartney, J. - Lawson R. - Hall J. (2016) Economic Freedom of the World 2016 Annual Report. Fraser Institute

\begin{tabular}{llll} 
& & I. & Tamaño del gobierno \\
A & Consumo del gobierno & D. & Tasa marginal máxima \\
\hline B & Transferencias y subsidios & (i) & $\begin{array}{l}\text { Tasa de impuesto sobre la renta marginal } \\
\text { superior }\end{array}$ \\
\hline C & Empresas e inversión pública & (ii) & $\begin{array}{l}\text { Tasa de impuesto sobre el ingreso marginal superior } \\
\text { y nóminas }\end{array}$ \\
\hline
\end{tabular}

las políticas y las instituciones de los países apoyan la libertad económica. Es así que el Índice de Libertad Económica se compone de cinco áreas generales (escala de 1 a 10): I) Tamaño del gobierno, II) Estructura legal, III) Moneda sana, IV) Libertad de comercio internacional, V) Regulación. Dentro de este marco, el presente estudio aborda la temática del área I), esto es, el tamaño del gobierno. En ese sentido, tal como se sostiene en el EFW, los elementos de esta área indican el grado en que los países confían en el proceso político para asignar recursos y bienes y servicios. Cuando el gasto del gobierno aumenta en relación con el gasto de los individuos, las familias y las empresas, la toma de decisiones políticas se substituye por la elección personal y la libertad económica se reduce.

Específicamente, mide el grado en que un país depende de la elección personal y los mercados en lugar de los presupuestos del gobierno y la toma de decisiones políticas. Por lo tanto, los países con bajos niveles de gasto público como porcentaje del total, un menor sector empresarial público y menores tasas de impuestos marginales obtienen las calificaciones más altas en esta zona.

Tal como se aprecia en el siguiente Cuadro, se utilizan cuatro (4) componentes para la construcción del índice del tamaño del gobierno: 


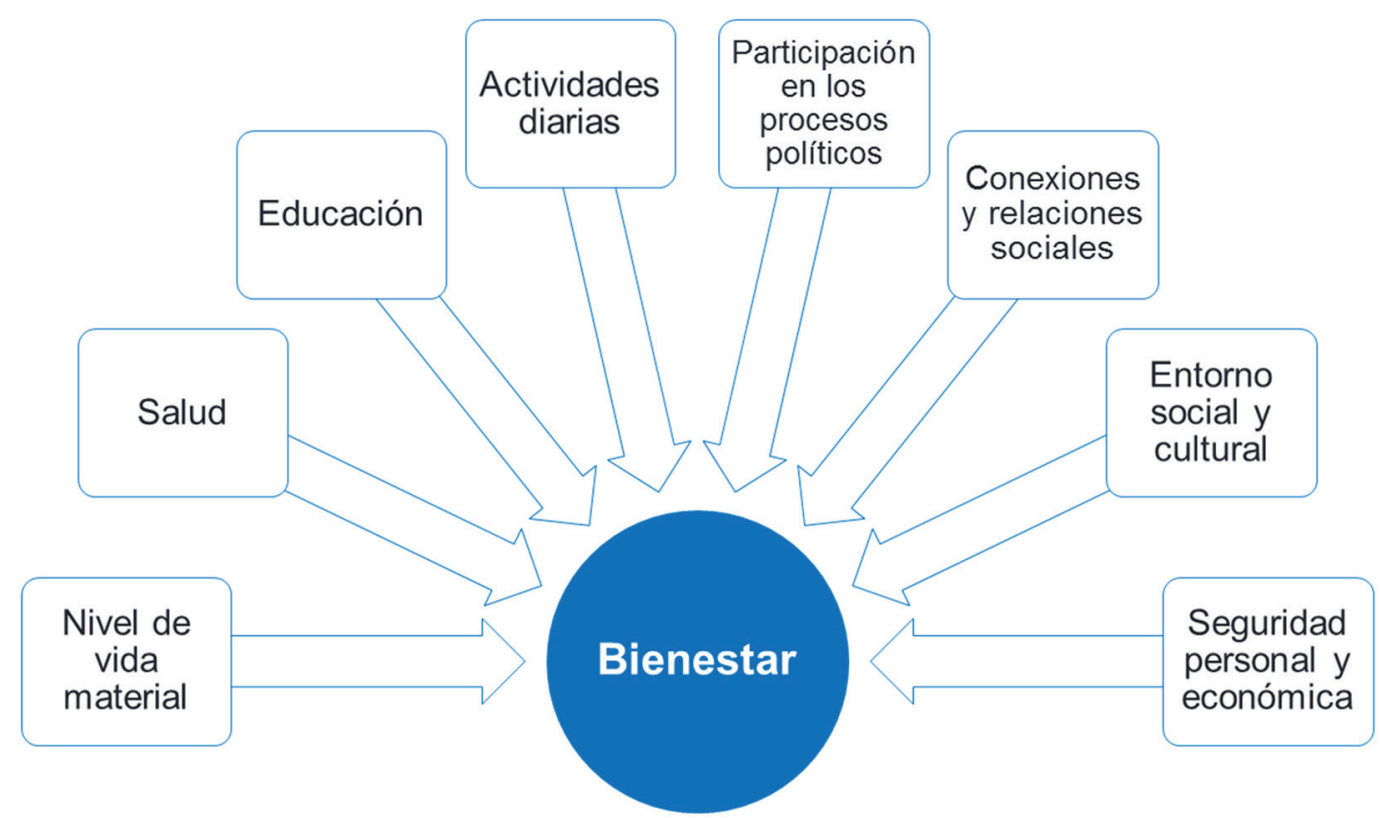

Figura 1. Dimensiones del bienestar. Fuente: Stiglitz, Sen y Fitoussi (2013)

\section{Bienestar}

Como sostienen Stiglitz, Sen y Fitoussi (2013) para precisar lo que significa bienestar hay que utilizar una definición con muchas dimensiones. A partir de la investigación académica y de un buen número de iniciativas concretas desarrolladas en todo el mundo, la comisión ha identificado las siguientes facetas clave que deben tenerse en cuenta simultáneamente: a) nivel de vida material (ingresos, consumo y riqueza), b) salud, c) educación, d) actividades personales (incluido el trabajo), e) voz política (participar como ciudadano) y gobernanza (vigencia del Estado de Derecho), f) conexiones y relaciones sociales (la gente con más contactos sociales evaluará mejor su vida, ya que muchas de las actividades más placenteras implican socialización), g) entorno (condiciones presentes y futuras), h) inseguridad física y económica (p. 59).
Debe enfatizarse que los autores argumentan que la calidad de vida depende de la salud y la educación de las personas, sus actividades diarias (que incluyen el derecho a un trabajo y a una vivienda digna), su participación en los procesos políticos, el entorno social y natural en el que viven y los factores que dan forma a su seguridad personal y económica. Lo que realmente importa son las capacidades de las personas, es decir, las oportunidades a su alcance y su libertad de elección.

No obstante lo anterior, un indicador que ha sido utilizado por mucho tiempo para medir el desarrollo relativo de un país es el Producto Bruto Interno (PBI) per cápita, el cual relaciona el PBI y la población de un país en un año determinado. Este indicador refleja la capacidad adquisitiva de los habitantes de un país. Pese a considerarse al PBI per cápita como un indicador del bienestar económico, presente algunos inconvenientes: 1) No reconoce las diferencias en la distribución del ingreso entre países; 2) Tiende a subestimar el nivel de vida de la población en aquellos países en los que la producción para el autoconsumo es una parte importante del total producido; 3) No toma en cuenta la conservación del medio ambiente o el aprovechamiento sostenible de los recursos naturales.

\section{MATERIAL Y MÉTODOS}

El estudio correspondió a un diseño no experimental pues no se manipuló la variable independiente.

La investigación fue descriptiva y correlacional. Descriptiva, ya que se centró en analizar la evolución de las variables objeto de estudio (tamaño del gobierno y PBI per cápita de los países miembros de la Alianza del Pacífco). Correlacional, porque tuvo como 
propósito determinar la relación que existe entre las variables antes señaladas. El enfoque fue cuantitativo por ser probatorio a través de mediciones estadísticas.

Datos del índice del tamaño del gobierno como componente del Índice de libertad económica y el Producto Bruto Interno (PBI) per cápita de los países de la Alianza del Pacífico para el período 1970-2014.

A partir de los datos obtenidos del índice del tamaño del gobierno como componente del Índice de Libertad Económica elaborado por el Instituto Fraser y los datos del PBI per cápita del Banco Mundial para los países de la Alianza del Pacífico, se recurrió al análisis descriptivo para examinar la evolución del tamaño del gobierno y el PBI per cápita y al análisis de pruebas estadísticas (ecuación de regresión, coeficiente de correlación y coeficiente de determinación) para establecer y calcular la relación entre las dos variables de interés.

El análisis de regresión consistió en recurrir al método de mínimos cuadrados, a través del cual se pudo construir los datos de "X" y "Y", esto es, la línea o ecuación que mejor representa la relación entre las dos variables de análisis (índice del tamaño del gobierno y PBI per cápita, en los países miembros de la Alianza del Pacífico). La ecuación general del método de los mínimos cuadrados que se emplea en el análisis de regresión es: $\mathbf{Y}=\mathbf{a}+\mathbf{b X}$.

De la observación de las variables durante el período 1970-2014, se derivó un diagrama de dispersión que permitió establecer la relación entre el índice del tamaño del gobierno y el bienestar individual en los países miembros de la Alianza del Pacífico (AP), tal como se aprecia en el siguiente figura.

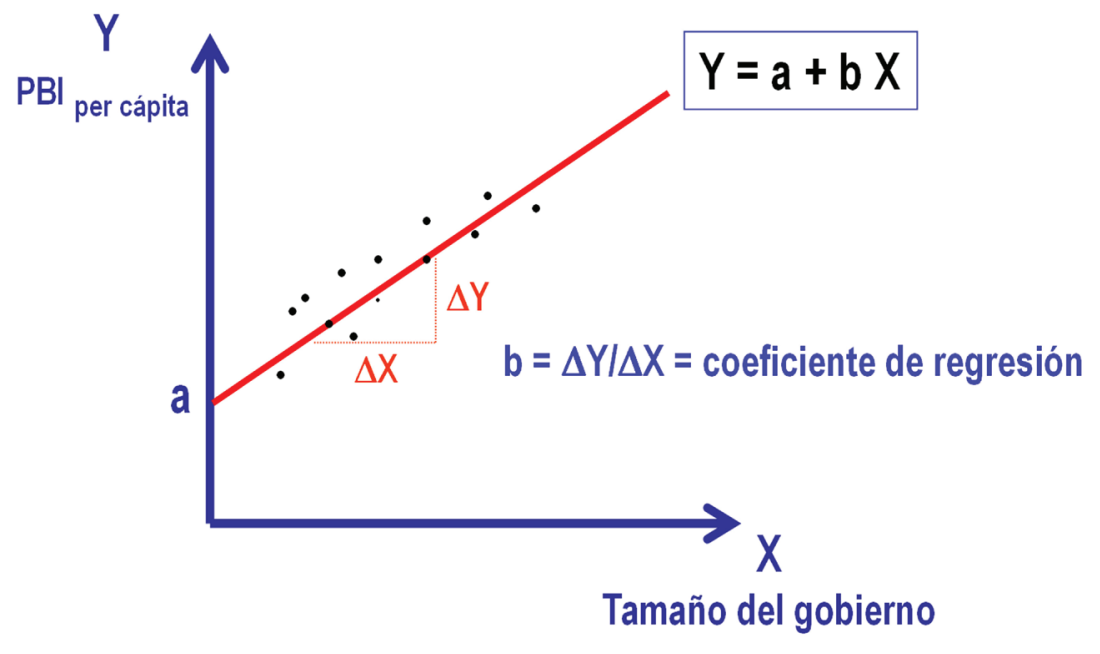

Figura 2. Ecuación de regresión. Fuente: Elaboración propia

Donde:

$\mathrm{Y}$ : variable dependiente (bienestar individual medido a través del PBI per cápita)

a : intersección estimada de la línea de regresión con el eje $\mathrm{Y}$

b : pendiente estimada de la línea de regresión: coeficiente de regresión

$\mathrm{X}$ : variable independiente (índice del tamaño del gobierno)

Luego, se determinaron los valores de "a" y "b". El método de los mínimos cuadrados permitió que la línea de regresión de mejor ajuste, reduzca al mínimo la suma de las desviaciones cuadráticas entre los valores reales y estimados de la variable dependiente para la información objeto de análisis; entonces se utilizaron las siguientes fórmulas para determinar los valores de "a"y "b":

$$
\begin{gathered}
b=\frac{(n)\left(\sum X Y\right)-\left(\sum X\right)\left(\sum Y\right)}{(n) \sum X^{2}-\left(\sum X\right)^{2}} \\
a=\bar{Y}-b \bar{X}
\end{gathered}
$$

Donde:

$\bar{Y}$ : Promedio de la variable $\mathrm{Y}$

$\bar{X}$ : Promedio de la variable $\mathrm{X}$

Seguidamente, se remplazaron los datos en la ecuación de mínimos cuadrados y se obtuvo la ecuación de regresión lineal para cada país miembro de la AP.

El análisis del coeficiente de correlación (r) sirvió para medir la fuerza o el grado de correlación entre las variables de interés en el análisis de regresión. La ecuación para medir el coeficiente de correlación es la siguiente:

$$
r=\frac{(n)\left(\sum X Y\right)-\left(\sum X\right)\left(\sum Y\right)}{\sqrt{\left[(n) \sum X^{2}-\left(\sum X\right)^{2}\right]\left[(n) \sum Y^{2}-\left(\sum Y\right)^{2}\right]}}
$$

Donde:

r : Coeficiente de correlación.

De manera que el coeficiente de correlación ( $r$ ) es un número que, en determinado conjunto de datos, se encuentra entre $[-1,1]$ y que indica: 
Dirección de la correlación. Si la correlación es positiva, significa que ambas variables ("X" y "Y") aumentan o disminuyen simultáneamente; por ejemplo, si " $X$ " aumenta, entonces "Y" tiende también a aumentar. Si la correlación es negativa, las variables tienden a moverse en direcciones opuestas; por tanto, si " $\mathrm{X}$ " aumenta, entonces " $Y$ " tiende a disminuir, o viceversa.

Fuerza de la relación. Cuanto mayor es el valor absoluto de $r$, más estrecha es la relación de las dos variables de estudio y mejor ajusta los datos el diagrama de dispersión de la ecuación de mínimos cuadrados. En los extremos

\section{Tabla 2.}

Tamaño del gobierno y PBI per cápita en países de la Alianza del Pacífico 19702014

Fuente: Fraser Institute, Banco Mundial / Elaboración propia

\begin{tabular}{rcccccrrr}
\multirow{2}{*}{ Año } & \multicolumn{3}{c}{ Índice del tamaño del gobierno } & \multicolumn{5}{c}{ PBI per cápita } \\
& Chile & Colombia & Perú & México & Chile & Colombia & México & Perú \\
\hline 1970 & 5,05 & 6,22 & 7,65 & 7,63 & 937 & 326 & 683 & 557 \\
\hline 1971 & 3,96 & 6,96 & 6,29 & 6,08 & 693 & 529 & 1446 & 1108 \\
\hline 1980 & 5,00 & 5,35 & 6,47 & 5,69 & 2454 & 1204 & 2803 & 1045 \\
\hline 1985 & 5,71 & 5,94 & 5,42 & 6,19 & 1362 & 1125 & 2386 & 847 \\
\hline 1990 & 6,51 & 7,23 & 7,13 & 7,96 & 2402 & 1175 & 3069 & 1210 \\
\hline 1995 & 7,30 & 6,83 & 8,21 & 7,47 & 5027 & 2471 & 3641 & 2218 \\
\hline 2000 & 6,12 & 4,64 & 8,07 & 7,14 & 5229 & 2472 & 6650 & 1997 \\
\hline 2001 & 7,11 & 4,78 & 7,87 & 7,38 & 4710 & 2396 & 6952 & 1981 \\
\hline 2002 & 7,09 & 4,73 & 7,55 & 7,36 & 4567 & 2356 & 7024 & 2059 \\
\hline 2003 & 7,28 & 4,85 & 7,63 & 7,30 & 4949 & 2246 & 6673 & 2180 \\
\hline 2004 & 7,34 & 5,29 & 7,79 & 7,33 & 6324 & 2740 & 7115 & 2448 \\
\hline 2005 & 7,43 & 4,45 & 7,71 & 7,11 & 7729 & 3386 & 7894 & 2755 \\
\hline 2006 & 7,50 & 4,90 & 7,73 & 7,08 & 9501 & 3709 & 8666 & 3171 \\
\hline 2007 & 7,95 & 5,57 & 7,79 & 7,43 & 10514 & 4674 & 9223 & 3611 \\
\hline 2008 & 7,88 & 5,79 & 7,85 & 7,12 & 10791 & 5434 & 9579 & 4209 \\
\hline 2009 & 7,78 & 6,29 & 7,51 & 6,79 & 10217 & 5148 & 7661 & 4166 \\
\hline 2010 & 7,91 & 6,16 & 7,53 & 7,18 & 12785 & 6251 & 8861 & 5021 \\
\hline 2011 & 8,00 & 6,20 & 7,56 & 7,02 & 14582 & 7228 & 9730 & 5770 \\
\hline 2012 & 8,00 & 6,06 & 7,59 & 7,10 & 15253 & 7885 & 9721 & 6386 \\
\hline 2013 & 8,03 & 6,32 & 746 & 7,09 & 15765 & 8031 & 10199 & 6581 \\
\hline 2014 & 7,99 & 6,09 & 7,40 & 7,80 & 14566 & 7918 & 10353 & 6490 \\
\hline & & & & & & & & \\
\hline
\end{tabular}
las variables
El coeficiente de determinación $\left(\mathrm{R}^{2}\right)$ representa la proporción de la variación total en la variable $\mathrm{Y}$, que se explica por la ecuación de regresión, pudiendo tomar un valor entre 0 y 1 . Es el cuadrado del coeficiente de correlación y asume un significado especial porque su valor representa la proporción de la variación de la variable dependiente $\mathrm{Y}$ que se explica por la variable independiente $\mathrm{X}$ mediante la ecuación de regresión.

$$
\mathrm{R}^{2}=\mathrm{r} \times \mathrm{r}
$$

Si $\mathrm{r}=0 \rightarrow$ no existe relación lineal entre

Donde:

r : Coeficiente de correlación.

$\mathrm{R}^{2}$ : Coeficiente de determinación.

\section{RESULTADOS}

El Reporte Anual del Economic Freedom of The World (EFW) publicado por Gwartney, Lawson y Hall (2016) es un instrumento valioso no sólo para medir la libertad económica de un país a través del tiempo, la cual toma en consideración diversos aspectos que contribuyen a explicar la libertad. También permite realizar investigaciones acerca de los efectos de la libertad económica sobre diversas variables de resultados, entre las cuales se encuentra el bienestar de quienes la experimentan o carecen de ella.

En la tabla 2 se observa la evolución de las variables dependiente e independiente que se utilizaron para el análisis de las pruebas estadísticas.

Análisis del tamaño del gobierno en países de la Alianza del Pacífico

Los datos del EFW permitieron analizar la evolución del índice del tamaño del gobierno en los países miembros de la Alianza del Pacífico. Como se 

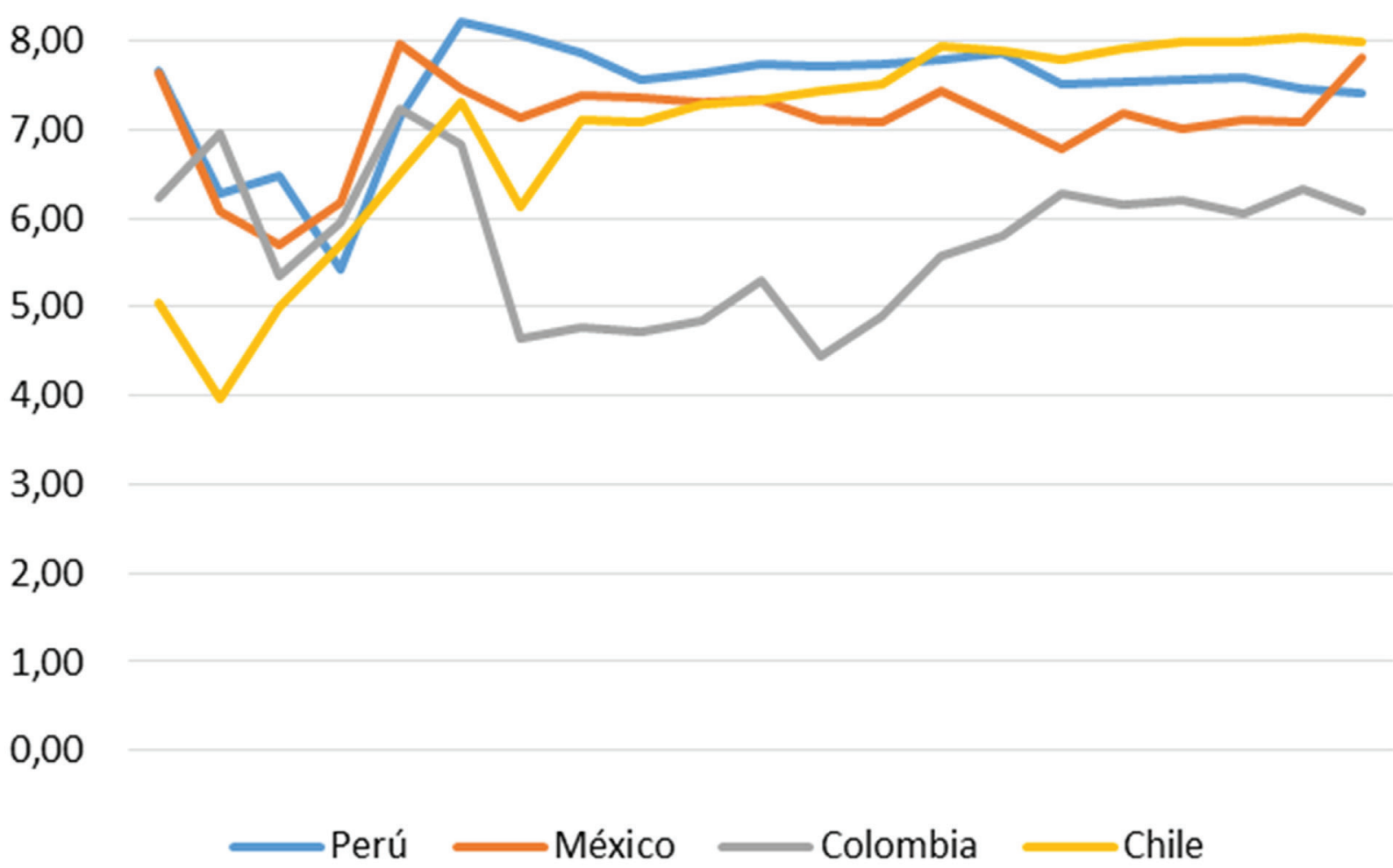

Figura 3. Evolución del índice del tamaño del gobierno en países de la Alianza del Pacífico 1970-2014 Fuente: Fraser Institute / Elaboración propia

observa en el Figura. 3, entre los años 1970 y 2014, el grado de libertad del tamaño del gobierno de Perú, México y Chile siguió un similar trayecto, excepto Colombia; destacando el período comprendido entre 1990 y 1995, donde los países miembros de la Alianza del Pacífico adoptaron diversas reformas en materia económica entre las que destacaron las finanzas públicas sanas (disciplina en las cuentas fiscales), control sobre el gasto público y emisiones monetarias (disciplina en las cuentas monetarias), incentivos a las inversiones nacionales y extranjeras, liberalización de los mercados y subsidios mínimos. No obstante ello, Colombia presentó una significativa caída en la calificación del tamaño del gobierno a partir del año 2010 y recién se recuperó en el 2009.

Análisis del grado de relación entre el tamaño del gobierno y el bienestar en países de la Alianza del Pacífico (AP)

El principal resultado obtenido es que existen diferencias en el grado de relación entre el índice del tamaño del gobierno y el PBI per cápita entre los países miembros de la Alianza del $\mathrm{Pa}$ cífico (AP). Tal como se observa en el Figura 4, en el período 1970-2014 el grado de relación entre las variables de análisis difiere entre los países de la AP, denotando una relación positiva y significativa solamente en el caso de Chile, cuyo grado de correlación es $r$ $=+0,83$; seguido por Perú $(r=+0,32)$, México $(\mathrm{r}=+0,31)$ y Colombia $(\mathrm{r}=$ $+0,11)$. 

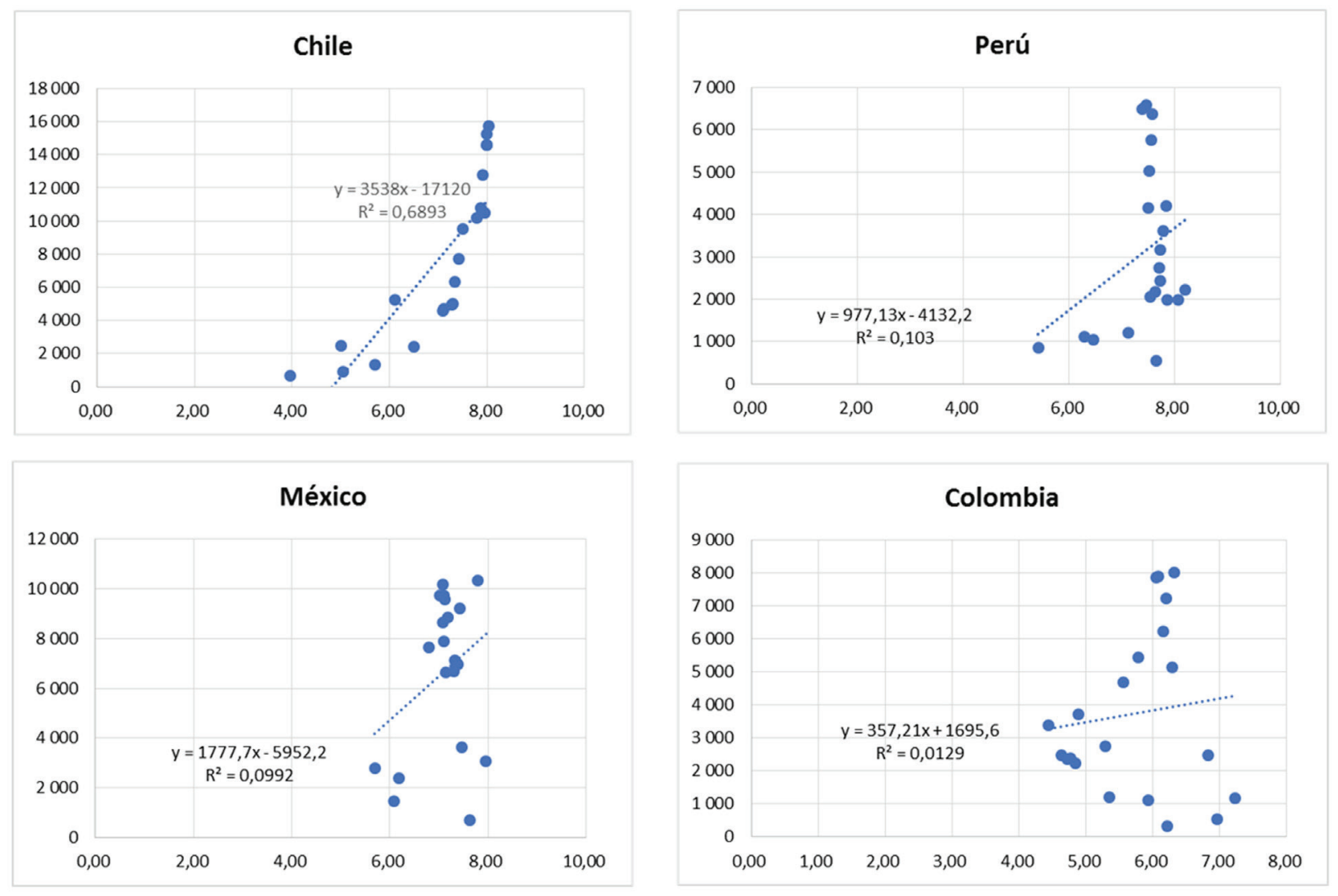

Figura 4. Relación entre el tamaño de gobierno y PBI per cápita en países de la AP 1970-2014 1970-2014

Fuente: Fraser Institute, Banco Mundial / Elaboración propia

En virtud de que el valor de $\mathrm{r}$ calculado para el caso de Chile es positivo $(+0,83)$, la relación entre el índice del tamaño del gobierno y el PBI per cápita es directa, esto es, cuando aumenta el índice del tamaño del gobierno aumenta el PBI per cápita. Asimismo, como el valor de $\mathrm{r}=+0,83$ se acerca al valor +1 , significa que hay una relación muy estrecha entre ambas variables.

Lo señalado en el párrafo precedente para el caso de Chile, se puede evidenciar a través del valor obtenido en el coeficiente de determinación $\mathrm{R}^{2}=0,6893$, lo cual significa que el $68,93 \%$ de la varianza en el PBI per cápita se explica por los cambios en el índice del tamaño del gobierno. La otra parte de la varianza en el PBI per cápita $(31 \%)$, se debe a otros factores diferentes del cambio en el índice del tamaño del gobierno.

Del mismo modo, en los casos de Perú $(\mathrm{r}=+0,32)$ y México $(\mathrm{r}=+0,31)$, si bien la relación entre el índice del tamaño del gobierno y el PBI per cápita es positiva, no existe una relación muy estrecha entre las dos variables de interés, tal como se observaron en sus coeficientes de determinación, cuyos valores son menores a 0,103 .

En el caso de Colombia $(r=+0,11)$ la relación entre ambas variables es la menor de los cuatro países de la AP, y consiguientemente, la relación es menos estrecha, lo cual se corrobora con el coeficiente de determinación $\mathrm{R} 2=$ 0,0129 , esto es, solamente el $1 \%$ de la varianza en el PBI per cápita se explica por los cambios en el índice del tamaño del gobierno. Con lo cual, la otra parte de la varianza en el PBI per cápita (99\%), se debe a otros factores diferentes del cambio en el índice del tamaño del gobierno.

\section{DISCUSIÓN}

La investigación desarrollada no cuenta con antecedentes directos sobre las variables de interés, ya que los estudios existentes fundamentalmente abordan el bienestar social y la desigualdad de ingresos a diferencia de otros que analizan el tamaño del gobierno medido a través de la repre- 
sentatividad del ingreso y gasto público respecto del PBI. Por tal razón, el presente estudio resulta muy relevante debido a que se enfoca en la relación particular entre el índice del tamaño del gobierno como componente del índice de libertad económica y el PBI per cápita en los países miembros de la Alianza del Pacífico (AP).

Siendo así, los resultados del grado de relación entre el índice del tamaño del gobierno y el PBI per cápita entre los países de la Alianza del Pacífico durante el período 1970-2014, fueron los siguientes: Chile $(\mathrm{r}=+0,83)$, Perú $(\mathrm{r}=$ $+0,32)$, México $(r=+0,31)$ y Colombia $(\mathrm{r}=+0,11)$.

En estricto, Chile tiene el mejor desempeño en la calificación del índice del tamaño del gobierno, alcanzando su nivel más alto en el año $2013(8,03)$. Precisamente, el tamaño del gobierno de nuestro país en el año $2014(7,40)$ empeora ligeramente si se compara con el $2013(7,46)$, es más aún no se recupera de los niveles obtenidos en 1995. Así, el Perú se encuentra un poco estancado respecto de la medición del año 1995, donde alcanzó el nivel más alto $(8,21)$ del período 1970-2014.

Ahora bien, si bien la teoría de la intervención del Rothbard es bastante extrema al considerar que toda intervención del gobierno es ilegal, nos permite comprender que la mayor calificación en el índice del tamaño del gobierno está asociado con la mayor libertad de elegir de los agentes que participan en el mercado, lo cual en cierta forma implica mayor libertad económica. En cuanto a los enfoques del bienestar, pese a los inconvenientes que representa la comparación a través del PBI per cápita, termina siendo un indicador estándar que tiene al menos datos históricos necesarios para la comparación entre los diversos países.

Finalmente, en virtud que se requieren otros estudios relacionados con la temática abordada en la presente investigación, la agenda de investigación en el futuro debería tratar de responder las siguientes interrogantes: ¿En qué medida el tamaño de gobierno es fundamental para el crecimiento económico?, ¿Cómo influye la interacción de otros componentes del tamaño del gobierno en la libertad de elegir de los agentes económicos?, ¿Cuál es la relación que existe entre el índice del tamaño del gobierno y el PBI per cápita en países miembros de la OCDE?

El debate sobre la intervención del gobierno en la economía, medido fundamentalmente a través del tamaño del gobierno, viene tomando mayor relevancia en los últimos años. Es así que lo importante del estudio es la implicancia que tiene el tamaño del gobierno sobre la libertad de elegir de las personas, ya que la mayor intervención del gobierno distorsiona las decisiones de los diversos agentes económicos.

Durante el período 1970-2014, el grado de relación entre índice del tamaño del gobierno y el PBI per cápita es más elevado para el caso de Chile, seguido de Perú, México y Colombia. Siendo así, de acuerdo con los resultados obtenidos a través de las pruebas estadísticas se concluye que el tamaño de gobierno es una condición necesaria pero no suficiente para determinar el bienestar individual entre los países de la Alianza del Pacífico. En efecto, esta no es la única razón, ya que existen otras variables que explican el bienestar de las personas.

Los fundamentos teóricos de la inter- vención del gobierno han sido fundamentales para comprender la importancia de los datos obtenidos del EFW, específicamente los datos de la calificación del índice del tamaño del gobierno en los países miembros de la Alianza del Pacífico. Sin embargo, al no existir antecedentes directos con el tema investigado, el presente estudio establece las bases para futuras investigaciones relacionadas con el tamaño del gobierno y el PBI per cápita.

\section{REFERENCIAS BIBLIOGRÁFICAS}

Gwartney, J., Lawson, R. \& Hall, J. (2016). Economic Freedom of the World 2016 Annual Report. Vancouver: Fraser Institute.

Krugman, P., Wells, R. (2013). Microeconomía. (3ra ed.). Barcelona: Editorial Reverté S.A.

Mises, L. (2011). La acción humana. (10ma ed.). Madrid. Unión Editorial S.A.

Rothbard, M. (2015). Poder y mercado. Guatemala: Unión Editorial S.A.

Rodríguez, V. (2016). Libertad económica y bienestar individual en Perú. Quipukamayoc, 24(46), 25-36.

Rodríguez, V. (2013). Fallas del mercado y regulación económica:¿la regulación ejercida por el gobierno puede permite lograr un mejor funcionamiento de los mercados. Quipukamayoc, 21 (39), 99-111.

Stiglitz, J., Rosengard, J. (2016). La economía del sector público. (4ta ed.). Barcelona: Editorial Antoni Bosch.

Stiglitz, J., Sen, A. \& Fitoussi, J. (2013). Medir nuestras vidas. Las limitaciones del PIB como indicador de progreso. Barcelona: RBA Libros S.A. 